BMJ Open

Diabetes

Research

\& Care

\title{
Glucagon-like peptide-1 receptor agonists are not associated with retinal adverse events in the FDA Adverse Event Reporting System
}

\author{
Gian Paolo Fadini, ${ }^{1}$ Mayur Sarangdhar, ${ }^{2}$ Angelo Avogaro ${ }^{3}$
}

\begin{abstract}
To cite: Fadini GP, Sarangdhar M, Avogaro A. Glucagon-like peptide-1 receptor agonists are not associated with retinal adverse events in the FDA Adverse Event Reporting System. BMJ Open Diab Res Care 2018;6:e000475. doi:10.1136/ bmjdrc-2017-000475
\end{abstract}

Received 11 September 2017 Revised 13 December 2017 Accepted 8 January 2018

\section{Check for updates}

${ }^{1}$ Department of Medicine, University of Padua, Padova, Italy

${ }^{2}$ Cincinnati Children's Hospital Medical Center, Cincinnati, Ohio, USA

${ }^{3}$ Department of Clinical and Experimental Medicine, University of Padua, Padova Italy

Correspondence to Professor Gian Paolo Fadini; gianpaolo.fadini@unipd.it

\section{ABSTRACT}

Objectives Glucagon-like peptide-1 receptor agonists (GLP-1RA) are widely used for the treatment of type 2 diabetes. In large trials, the GLP-1RAs liraglutide and semaglutide improved cardiovascular outcomes, but semaglutide was associated with an increased risk of retinopathy progression. We herein evaluated the association between GLP-1RA and retinal adverse events (AE) in the Food and Drug Administration Adverse Event Reporting System (FAERS).

Research design and methods We mined the FAERS between 2004q1 and 2017q1 (for a total of 9217555 $A E$ reports) to analyze disproportionality and evaluate the association between GLP-1RAs and AEs involving the retina. We compared the frequency of retinal AEs among reports including GLP-1RAs and in those including other glucose-lowering medications (GLMs) as suspect or concomitant drugs.

Results We retrieved 114814 reports involving GLP-1RA and 694725 reports involving other GLMs as suspect or concomitant drugs. The cumulative frequency of retinal AEs was 2.53/1000 for reports involving GLP-1RA vs $6.62 / 1000$ for reports involving other GLMs, with a proportional reporting ratio of $0.38(95 \% \mathrm{Cl} 0.34$ to 0.43 ; $\mathrm{P}<0.0001)$. Reports involving GLP-1RAs listed significantly more comorbid conditions and concomitant medications. Findings were consistent after filtering the diabetes indication irrespective of concomitant GLM, in reports including and in those not including insulin, and for the various GLP-1RAs.

Conclusions In the FAERS there is no evidence that GLP1RAs are associated with AEs suggestive of retinopathy progression. Despite more comorbid conditions and concomitant medications, in reports with GLP-1RA the frequency of retinal AEs was significantly lower than in reports with other GLMs.

\section{INTRODUCTION}

Glucagon-like peptide-1 receptor agonists (GLP-1RAs) are widely used for the treatment of type 2 diabetes. Thanks to their glycemic and extraglycemic effects, GLP-1RAs are expected to exert protective effects on chronic diabetic complications. ${ }^{1}$ In a postmarketing trial, the once-daily GLP-1RA

\section{Significance of this study}

What is already known about this subject?

- The glucagon-like peptide-1 receptor agonists (GLP-1RA) liraglutide and semaglutide improved cardiovascular outcomes in people with type 2 diabetes.

- In the SUSTAIN-6 trial, the GLP-1RA semaglutide was associated with an increased risk of retinopathy progression, but a meta-analysis rules out that GLP-1RA as a class increases the risk of retinopathy.

What are the new findings?

- We analyzed a pharmacovigilance database containing almost 10 million adverse event (AE) reports to evaluate the association between GLP-1RAs and retinal AEs and found no evidence that GLP-1RAs are associated with AEs suggestive of retinopathy progression.

- Despite more comorbid conditions and concomitant medications, the frequency of retinal AEs for GLP-1RAs was significantly lower than for other glucose-lowering medications.

How might these results change the focus of research or clinical practice?

- Our findings reassure the risk of retinopathy progression raised by the SUSTAIN- 6 trial.

- Other explanations for the increased retinopathy progression observed with semaglutide therapy need to be considered.

liraglutide significantly reduced cardiovascular events and mortality. ${ }^{2}$ In the premarketing SUSTAIN-6 trial, the once-weekly GLP-1RA semaglutide, which is structurally related to liraglutide, also significantly reduced cardiovascular events. ${ }^{3}$ Differently, the short-acting GLP-1RA lixisenatide and the once-weekly exenatide had a neutral effect on cardiovascular outcomes. ${ }^{45}$

Interestingly, both liraglutide and semaglutide showed evidence of renal protection, ${ }^{36}$ but semaglutide was associated with 
a significant $76 \%$ increased risk of retinopathy complications (vitreous hemorrhage, blindness, or conditions requiring treatment with an intravitreal agent or photocoagulation). ${ }^{3}$ A similar phenomenon was reported previously with other GLP-1RAs. ${ }^{7}$ The possible reasons for such unexpected findings in SUSTAIN-6 include issues related to trial design, the rapid improvement in glucose control, and a direct angiogenic or toxic effect of semaglutide. Of note, preclinical studies have shown that topical treatment with a GLP-1RA or a dipeptidyl peptidase-4 inhibitor protects from neurodegeneration in experimental diabetic retinopathy. ${ }^{910}$ These data argue against a direct adverse effect of GLP-1RA on retinopathy progression, but an eventual proangiogenic effect of semaglutide still needs to be ruled out.

Importantly, a recent meta-analysis of randomized controlled trials (RCTs) found that treatment with GLP1-RAs, as a class, was not associated with a significant increase in the incidence of retinopathy. ${ }^{11}$ Studies using routinely accumulated clinical data are useful to complement or challenge RCT findings. ${ }^{12}$ Since adverse event (AE) reporting is a routine duty of clinicians, pharmacovigilance studies belong to such category of 'real world studies'. We argue that, if GLP-1RA were truly associated with retinopathy progression, this should emerge as a safety signal from pharmacovigilance assessment, as it recently occurred for the association between canagliflozin and amputations. ${ }^{13} 14$ Thus, to evaluate the association between GLP-1RAs and retinopathy, we herein analyzed the Food and Drug Administration (FDA) Adverse Event Reporting System (FAERS), which is a global pharmacovigilance database used to monitor drugs' safety signals.

\section{RESEARCH DESIGN AND METHODS}

\section{Data source}

Pharmacovigilance databases can be used to assess a drug's safety by evaluating disproportional associations between the drug and one or more AEs. If there is no link between a drug and an AEs, the frequency of such AEs will be uniformly distributed in reports listing and in those not listing that drug as suspect or concomitant, without disproportionality. On the contrary, AEs that are caused by a drug will occur more frequently in reports listing than in those not listing that drug as suspect or concomitant, thus generating disproportionality. ${ }^{15}$

The FAERS database contains millions of AE reports filed to the FDA from all over the world. Each report contains a unique identifier, the name of suspect and concomitant drugs, their dosage, duration and indications for use, along with demographic characteristics of the patients, outcomes, and reporting source and country. However, not all AE reports contain complete data. FAERS files are made publicly available on a quarterly basis and need to be mined using sophisticated methods for orthogonal database search that are not routinely available to most clinical researchers.

\section{Queries}

To perform an unbiased analysis of disproportionality within the FAERS, we used AERSMine ${ }^{16}$ a web-based software, to mine the FAERS, which accesses files from 2004q1 to 2017q1, for a total of 9217555 reports. Queries were run on AERSMine to analyze disproportionality and evaluate the association between GLP-1RA and AEs involving the retina. In the first search strategy (model 1 ), we compared the frequencies of retinal AEs among reports listing GLP-1RA versus reports listing any other glucose-lowering medication (GLM) except GLP-1RA. To be more specific, in the second search strategy (model 2 ), we filtered only reports with an expanded diabetes indication. We retrieved the number and frequencies of each specific retinal $\mathrm{AE}$, as well as the total number of unique reports of at least one retinal AE. We also recorded concomitant medications grouped by therapeutic category and concomitant indications grouped by system organ class. Figure 1 illustrates the study query flow chart.

The following search strings were used for AERSMine queries:

GLP-1RA group: 'insulin degludec and liraglutide' or 'liraglutide' OR 'exenatide' OR 'lixisenatide' OR 'albiglutide' OR 'dulaglutide' OR 'teduglutide'.

Control group: 'insulins and analogues' OR 'insulins and analogues for injection, long-acting' OR 'insulins and analogues for injection, fast-acting' OR 'insulins and analogues for injection, intermediate- or long-acting combined with fast-acting' OR 'insulins and analogues for injection, intermediate-acting' OR 'insulin glargine' OR 'insulin (human)' OR 'insulins and analogues for inhalation' OR 'insulin lispro' OR 'insulin aspart' OR 'insulin detemir' OR 'insulin glulisine' OR 'insulin degludec' OR 'insulin (pork)' OR 'insulin (beef)' OR 'insulin degludec and insulin aspart' OR 'canagliflozin' OR 'dapagliflozin' OR 'empagliflozin' OR 'sitagliptin' OR 'saxagliptin' OR 'linagliptin' OR 'vildagliptin' OR 'alogliptin' OR 'metformin' OR 'glibenclamide' OR 'glimepiride' OR 'gliclazide' OR 'tolbutamide' OR 'chlorpropamide' OR 'glipizide' OR 'gliquidone' OR 'rosiglitazone' OR 'pioglitazone' OR 'troglitazone' OR 'acarbose' OR 'voglibose' OR 'repaglinide' OR 'nateglinide' OR 'mitiglinide'. Excluded: 'insulin degludec and liraglutide' or 'liraglutide' OR 'exenatide' OR 'lixisenatide' OR 'albiglutide' OR 'dulaglutide' OR 'teduglutide'.

Retinal AEs: 'diabetic retinopathy' OR 'retinal detachment' OR 'retinopathy' OR 'retinal haemorrhage' OR 'retinal vein occlusion' OR 'diabetic retinal oedema' OR 'retinal oedema' OR 'retinal disorder' OR 'retinal aneurysm' OR 'retinal exudates' OR 'retinal tear' OR 'retinal vascular thrombosis' OR 'retinal scar' OR 'retinal injury' OR 'retinopathy proliferative' OR 'retinopathy haemorrhagic' OR 'retinal vascular disorder' OR 'retinal artery occlusion' OR 'retinal degeneration' OR 'retinal operation' OR 'retinal ischaemia' OR 'retinogram abnormal' OR 'retinal artery embolism' OR 'retinal telangiectasia' 


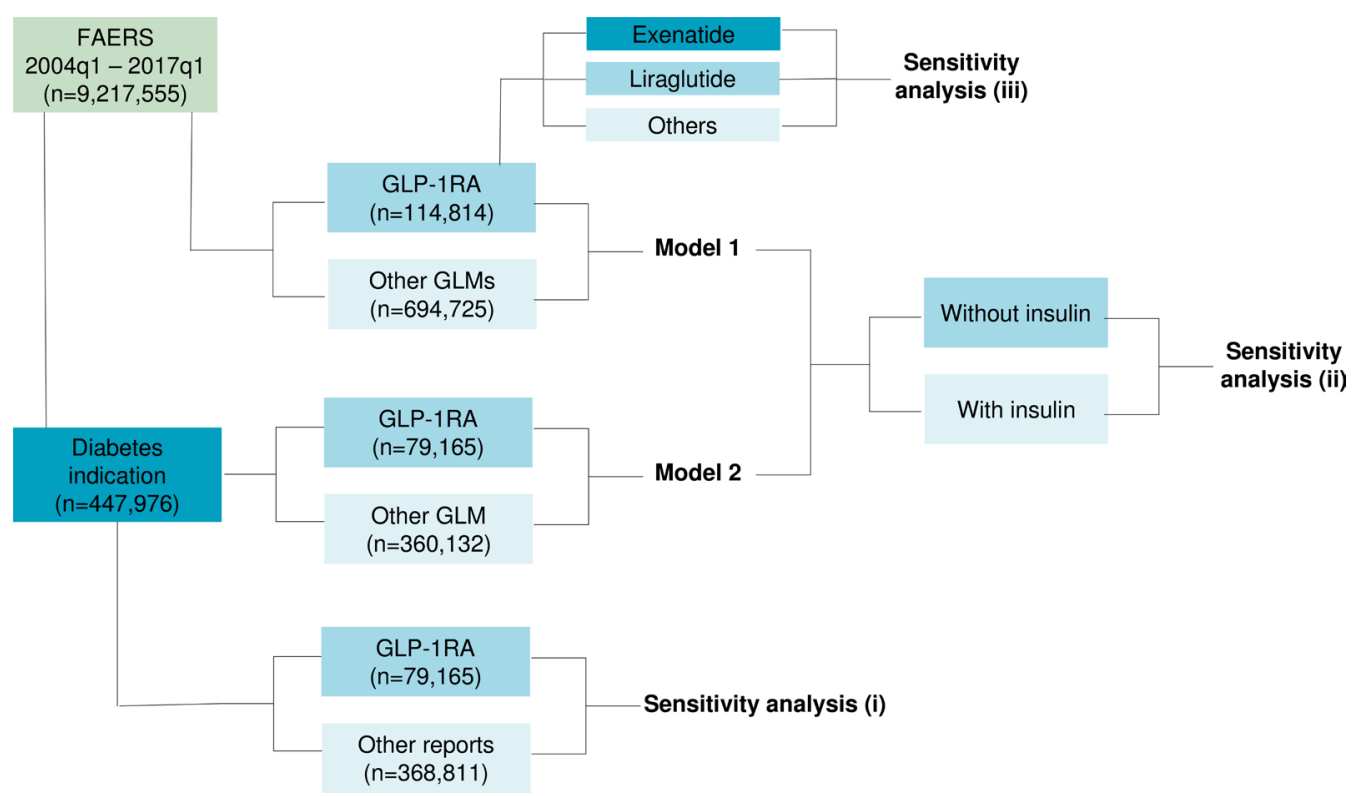

Figure 1 Study flow chart. Sequential queries of the FAERS, including subanalyses, are represented with the respective number of total reports. FAERS, Food and Drug Administration Adverse Event Reporting System; GLMs, glucose-lowering medications; GLP-1RA, glucagon-like peptide-1 receptor agonists.

OR 'retinal microaneurysms' OR 'retinal neovascularisation' OR 'retinopexy' OR 'retinal dystrophy' OR 'retinal laser coagulation' OR 'retinopathy background' OR 'retinal artery stenosis' OR 'angiogram retina abnormal'.

Blindness was examined separately using this query string: 'blindness' OR 'blindness unilateral' OR 'diabetic blindness'.

Diabetes indication: 'glucose metabolism disorders (incl diabetes mellitus)' OR 'diabetes mellitus (incl subtypes)' OR 'diabetes mellitus' OR 'type 2 diabetes mellitus' OR 'diabetes mellitus non-insulin-dependent' OR 'insulin-requiring type 2 diabetes mellitus' OR 'diabetes mellitus insulin-dependent' OR 'insulin-requiring type ii diabetes mellitus' OR 'diabetes mellitus inadequate control' OR 'diabetes mellitus management' OR 'diabetes' OR 'type ii diabetes mellitus' OR 'diabetes prophylaxis' OR 'insulin resistant diabetes' OR 'insulin-dependent diabetes mellitus' OR 'non-insulin-dependent diabetes mellitus' OR 'diabetes mellitus nos' OR 'diabetes mellitus loss of control' OR 'diabetes mellitus poor control' OR 'diabetes mellitus without mention of complication' OR 'diabetes steroid-induced' OR 'diabetes with renal manifestations'.

A custom procedure was implemented on AERSMine to retrieve the percentage of $\mathrm{AE}$ reports wherein the drugs of interest were indicated as primary suspect or concomitant.

In $\mathrm{AE}$ reports, including those filed to the FAERS, coding drug exposure time is optional and, as a result, only $\sim 30 \%$ of all reports contain suspect or concomitant drug starting date, which is the minimum information needed to calculate exposure time before the AE. Currently, no available software can automatically retrieve average exposure for queries of drug-AE combination. Thus, to calculate exposure time, we performed a manual mining of retinal AE reports including GLP-1RA as suspect or concomitant drug. We calculated exposure time in days as the time elapsed since the drug's starting date (when available) and ending date, or event date, or the date the manufacturer first received initial information, or the date the FDA received first version, whichever was available and occurred first.

\section{Sensitivity analyses}

In addition to the two major search strategies, we performed sensitivity analyses to challenge the robustness of the findings by modifying the search strategy and reporting the overall frequency of pooled retinal AEs, as follows: (1) by filtering the diabetes indication irrespective of other GLM; (2) by presence/absence of insulin as a concomitant drug (models 1 and 2); and (3) by GLP-1RA molecule (model 1).

\section{Statistical analysis}

For each search, we retrieved the total number of $\mathrm{AE}$ reports, the number of reports for each retinal $\mathrm{AE}$ in the two groups and the respective frequencies/1000 reports with 95\% CI. The proportional reporting ratio (PRR) was calculated as previously described. Statistical significance was accepted at $\mathrm{P}<0.05$, and the type I error inflation due to multiple testing was adjusted using Bonferroni correction.

\section{RESULTS}

\section{Data overview}

From 2004q1 to $2017 q 1$, the FAERS contained a total of 114814 unique $\mathrm{AE}$ reports listing one or more GLP-1RAs among suspect or concomitant drugs (1.25\% of FAERS reports). Distribution of individual GLP-1RAs in reports was as follows: exenatide $n=69754$ (60.8\%), liraglutide $\mathrm{n}=29738$ (25.9\%), albiglutide 


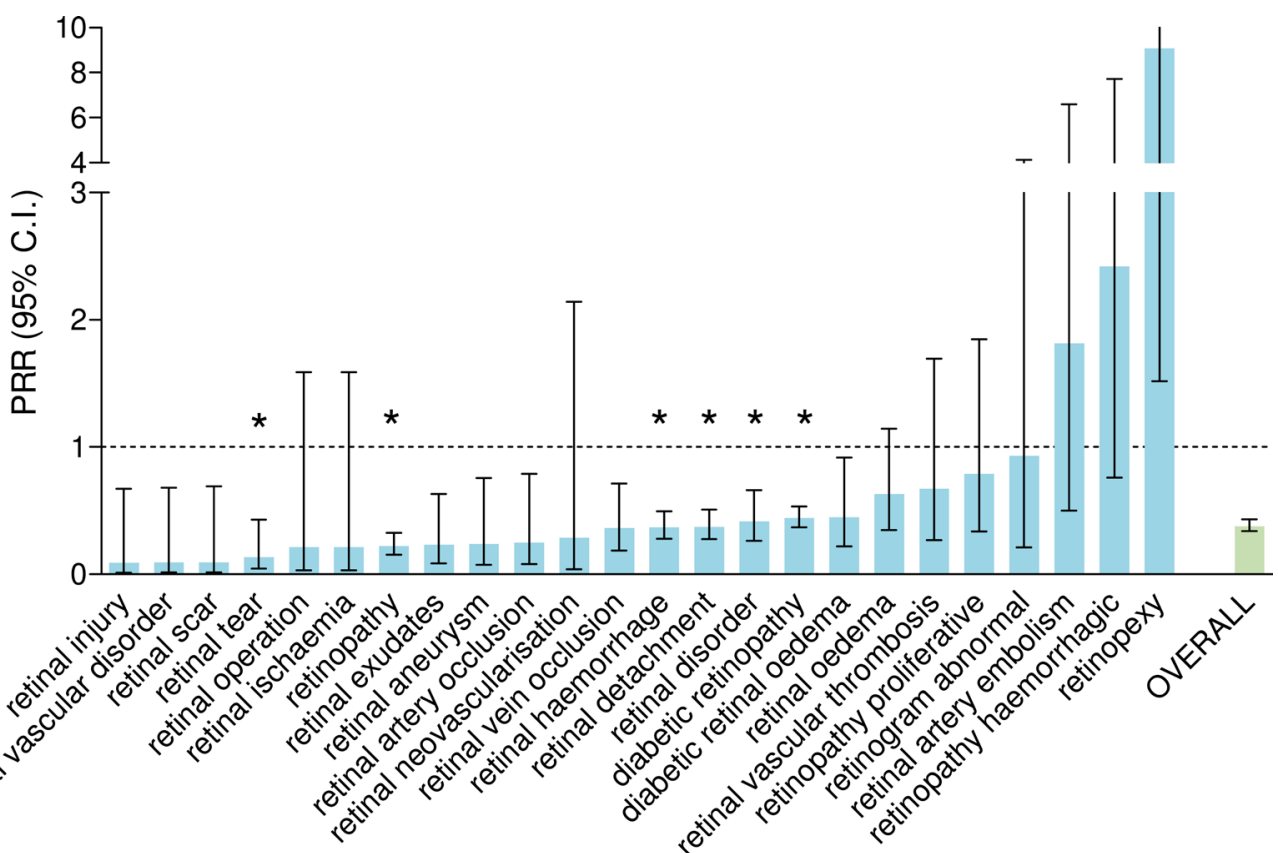

Figure 2 Disproportionality analysis for specific retinal adverse events. The proportional reporting ratios (PRR) are reported along with their $95 \% \mathrm{Cl}$ in model 1. *Significant after Bonferroni correction.

$\mathrm{n}=8028(7.0 \%)$, dulaglutide $\mathrm{n}=6971 \quad(6.1 \%)$, teduglutide $\mathrm{n}=1390(1.2 \%)$, and lixisenatide $\mathrm{n}=119(0.1 \%)$. Of the total of 114814 reports, $291(2.53 / 1000)$ included at least one retinal AE. GLP-1RAs were listed as suspect drugs in $55.8 \%$ of these reports. Of the 291 retinal AEs wherein a GLP-1RA was listed as suspect or concomitant, exposure was available for $81(27.8 \%)$ and was extremely variable, with a median of 124 days (IQR 29-276 days; range: 1 day to 10.2 years).

\section{Model 1}

Without filtering the diabetes indication (model 1), there were 694725 unique reports listing other GLMs, among which 4597 contained at least one retinal $\mathrm{AE}$ (6.62/1000). Thus, in model 1, the PRR for retinal AEs associated with GLP-1RAs was 0.38 (95\% CI 0.34 to $0.43 ; \mathrm{P}<0.0001)$. The percentage of retinal $\mathrm{AE}$ reports wherein a GLP-1RA or another GLM was listed as suspect was $55.8 \%$ vs $32.4 \%$, respectively $(\mathrm{P}<0.0001)$. Figure 2 and table 1 show the PRRs for 24 specific retinal AEs: 14/24 were significantly less frequent $(\mathrm{PRR}<1.0)$ while $1 / 24$ (retinopexy) was significantly more frequent $(\mathrm{PRR}>1.0)$ in reports with than in those without GLP-1RA. After applying Bonferroni correction, six retinal AEs occurred less frequently in reports with than in those without GLP-1RA (diabetic retinopathy, retinopathy, retinal hemorrhage, retinal disorder, retinal detachment, and retinal tear). Blindness was examined separately as it is not included among retinal AEs: its frequency was $0.83 / 1000$ in reports for GLP-1RA vs 4.03/1000 in reports for other GLMs, for a PRR of 0.21 (95\% CI 0.17 to $0.25 ; \mathrm{P}<0.0001)$.

We then checked the imbalance of concomitant drug and indication frequencies in reports listing versus those not listing GLP-1RAs. For most therapeutic drug classes where an imbalance was noted, a larger number of drugs were significantly more frequent in reports listing GLP-1RA than in control reports, including those for the cardiovascular system and metabolism (figure 3A). Similarly, for most system organ indication classes where an imbalance was noted, a larger number of conditions were significantly more frequent in reports with GLP-1RAs, including endocrine, metabolism, and cardiac disorders (figure 3B).

\section{Model 2}

We then filtered the query for an expanded diabetes indication and identified 79165 reports with GLP-1RAs, 214 of which contained at least one retinal $\mathrm{AE}(2.70 / 1000)$, and 360132 reports with other GLMs, 2528 of which contained at least one retinal AE (7.02/1000). Thus, in model 2 the PRR for retinal AEs associated with GLP-1RAs was 0.39 (95\% CI 0.34 to 0.44; $\mathrm{P}<0.0001)$. In model 2 , the percentage of retinal AE reports wherein a GLP-1RA or another GLM was listed as suspect was $69.0 \%$ vs $51.7 \%$, respectively $(\mathrm{P}<0.0001)$.

Table 1 shows that $8 / 23$ specific retinal AEs were significantly less frequent $(\mathrm{PRR}<1.0)$ while $1 / 23$ $\mathrm{AE}$ (retinopexy) was significantly more frequent in reports with than in those without GLP-1RAs. After Bonferroni correction, four retinal AEs showed a PRR significantly $<1.0$ for GLP-1RAs (retinopathy, diabetic retinopathy, retinal hemorrhage, and retinal detachment). The frequency of blindness was $1.20 / 1000$ in reports for GLP-1RA vs 7.78/1000 in reports for other GLMs, for a PRR of 0.15 (95\% CI 0.13 to 0.19 ; $\mathrm{P}<0.0001)$. 
Table 1 Association between GLP-1RA and retinal AEs in the FAERS

\begin{tabular}{|c|c|c|c|c|c|c|}
\hline \multirow[b]{2}{*}{$\begin{array}{l}\text { Adverse event, } n \\
(/ 1000)\end{array}$} & \multicolumn{3}{|l|}{ Model 1} & \multicolumn{3}{|l|}{ Model 2} \\
\hline & $\begin{array}{l}\text { GLP-1RA } \\
(n=114814)\end{array}$ & $\begin{array}{l}\text { Control } \\
(n=694725)\end{array}$ & PRR (95\% Cl) & $\begin{array}{l}\text { GLP-1RA } \\
(n=79165)\end{array}$ & $\begin{array}{l}\text { Control } \\
(n=360132)\end{array}$ & PRR (95\% Cl) \\
\hline Retinal injury & $1(0.01)$ & $65(0.09)$ & 0.09 (0.01 to 0.67$)$ & $1(0.01)$ & $40(0.11)$ & 0.11 (0.02 to 0.83$)$ \\
\hline $\begin{array}{l}\text { Retinal vascular } \\
\text { disorder }\end{array}$ & $1(0.01)$ & $64(0.09)$ & 0.09 (0.01 to 0.68$)$ & $0(0.00)$ & $27(0.07)$ & $\mathrm{N} / \mathrm{D}$ \\
\hline Retinal scar & $1(0.01)$ & $63(0.09)$ & 0.10 (0.01 to 0.69$)$ & $1(0.01)$ & $29(0.08)$ & $0.16(0.02$ to 1.15$)$ \\
\hline Retinal tear & $3(0.03)$ & $133(0.19)$ & $0.14(0.04 \text { to } 0.43)^{*}$ & $1(0.01)$ & $56(0.16)$ & 0.08 (0.01 to 0.59$)$ \\
\hline Retinal operation & $1(0.01)$ & $28(0.04)$ & $0.22(0.03$ to 1.59$)$ & $1(0.01)$ & $19(0.05)$ & 0.24 (0.03 to 1.79$)$ \\
\hline Retinal ischemia & $1(0.01)$ & $28(0.04)$ & 0.22 (0.03 to 1.59$)$ & $1(0.01)$ & $14(0.04)$ & $0.32(0.04$ to 2.47$)$ \\
\hline Retinopathy & $28(0.24)$ & $760(1.09)$ & $0.22(0.15 \text { to } 0.33)^{\star}$ & $23(0.29)$ & $427(1.19)$ & $0.25(0.16 \text { to } 0.37)^{*}$ \\
\hline Retinal exudates & $4(0.03)$ & $104(0.15)$ & 0.23 (0.09 to 0.63$)$ & $4(0.05)$ & $47(0.13)$ & $0.39(0.14$ to 1.07$)$ \\
\hline Retinal aneurysm & $3(0.03)$ & $76(0.11)$ & 0.24 (0.08 to 0.76$)$ & $3(0.04)$ & $40(0.11)$ & 0.34 (0.11 to 1.10$)$ \\
\hline Retinal artery occlusion & $3(0.03)$ & $73(0.11)$ & 0.25 (0.08 to 0.79$)$ & $2(0.03)$ & $26(0.07)$ & 0.35 (0.08 to 1.47$)$ \\
\hline $\begin{array}{l}\text { Retinal } \\
\text { neovascularization }\end{array}$ & $1(0.01)$ & $21(0.03)$ & $0.29(0.04$ to 2.14$)$ & $1(0.01)$ & $7(0.02)$ & 0.65 (0.08 to 5.28$)$ \\
\hline Retinal vein occlusion & $9(0.08)$ & $150(0.22)$ & $0.36(0.19$ to 0.71$)$ & $7(0.09)$ & $68(0.19)$ & 0.47 (0.22 to 1.02$)$ \\
\hline Retinal hemorrhage & $49(0.43)$ & $800(1.15)$ & $0.37(0.28 \text { to } 0.49)^{*}$ & $19(0.24)$ & $376(1.04)$ & $0.23(0.14 \text { to } 0.36)^{*}$ \\
\hline Retinal detachment & $43(0.37)$ & $697(1.00)$ & $0.37(0.27 \text { to } 0.51)^{\star}$ & $27(0.34)$ & $405(1.12)$ & $0.30(0.21 \text { to } 0.45)^{\star}$ \\
\hline Retinal disorder & $19(0.17)$ & $277(0.40)$ & $0.42(0.26 \text { to } 0.66)^{\star}$ & $17(0.21)$ & $158(0.44)$ & $0.49(0.30$ to 0.81$)$ \\
\hline Diabetic retinopathy & $122(1.06)$ & $1663(2.39)$ & $0.44(0.37 \text { to } 0.53)^{\star}$ & $92(1.16)$ & $960(2.67)$ & $0.44(0.35 \text { to } 0.54)^{\star}$ \\
\hline Diabetic retinal edema & $8(0.07)$ & $108(0.16)$ & 0.45 (0.22 to 0.92$)$ & $3(0.04)$ & $47(0.13)$ & 0.29 (0.09 to 0.93$)$ \\
\hline Retinal edema & $12(0.10)$ & $115(0.17)$ & 0.63 (0.35 to 1.14$)$ & $9(0.11)$ & $57(0.16)$ & 0.72 (0.36 to 1.45$)$ \\
\hline $\begin{array}{l}\text { Retinal vascular } \\
\text { thrombosis }\end{array}$ & $5(0.04)$ & $45(0.06)$ & 0.67 (0.27 to 1.69$)$ & $5(0.06)$ & $21(0.06)$ & $1.08(0.41$ to 2.87$)$ \\
\hline $\begin{array}{l}\text { Retinopathy } \\
\text { proliferative }\end{array}$ & $6(0.05)$ & $46(0.07)$ & 0.79 (0.34 to 1.85$)$ & $3(0.04)$ & $31(0.09)$ & 0.44 (0.13 to 1.44$)$ \\
\hline Retinogram abnormal & $2(0.02)$ & $13(0.02)$ & $0.93(0.21$ to 4.13$)$ & $2(0.03)$ & $4(0.01)$ & $2.27(0.42$ to 12.42$)$ \\
\hline Retinal artery embolism & $3(0.03)$ & $10(0.01)$ & $1.82(0.50$ to 6.60$)$ & $3(0.04)$ & $3(0.01)$ & 4.55 (0.92 to 22.54) \\
\hline $\begin{array}{l}\text { Retinopathy } \\
\text { hemorrhagic }\end{array}$ & $4(0.03)$ & $10(0.01)$ & $2.42(0.76$ to 7.72$)$ & $4(0.05)$ & $5(0.01)$ & $3.64(0.98$ to 13.55$)$ \\
\hline Retinopexy & $3(0.03)$ & $2(0.00)$ & 9.08 (1.52 to 54.32$)$ & $3(0.04)$ & $1(0.00)$ & 13.7 (1.4 to 131.2 ) \\
\hline Pooled & $291(2.53)$ & 4597 (6.62) & 0.38 (0.34 to 0.43$)$ & $214(2.70)$ & $2528(7.02)$ & 0.39 (0.34 to 0.44$)$ \\
\hline
\end{tabular}

Number of reports, rates/1000 reports, and PRR (with 95\% Cls) are shown for model 1 (no diabetes indication) and model 2 (filtered by diabetes indication).

*Significant after Bonferroni correction.

AE, adverse event; FAERS, Food and Drug Administration Adverse Event Reporting System; GLP-1RA, glucagon-like peptide-1 receptor agonists; N/D, not determined; PRR, proportional reporting ratio.

\section{Sensitivity analyses}

Diabetes indication filter without GLMs

In this analysis, we retrieved reports where the diabetes indication was included, listing or not listing GLP-1RAs irrespective of the presence of other GLMs. The retrieved data set was almost identical to model 2, with an overall PRR for retinal AEs associated with GLP-1RAs of 0.39 (95\% CI 0.34 to $0.44 ; \mathrm{P}<0.0001)$. Figure $4 \mathrm{~A}$ shows the overall PRR compared with model 1 and model 2.

\section{Presence/absence of insulin}

We performed separate subanalyses for model 1 and model 2 including only reports listing insulin as a concomitant drug or excluding reports listing insulin. Figure 4B shows no substantial difference of PRRs for retinal AEs associated with GLP-1RAs between reports with or without concomitant insulin therapy in model 1 . In model 2, the PRR was significantly lower in reports listing insulin as a concomitant drug.

\section{GLP-1RA molecule}

We performed separate subanalyses of model 1 by type of GLP-1RA. As albiglutide, dulaglutide, teduglutide, and lixisenatide had very small number of reports, they were pooled together. Figure 4C shows that the PRR for retinal AEs was quite similar for exenatide and liraglutide, the 

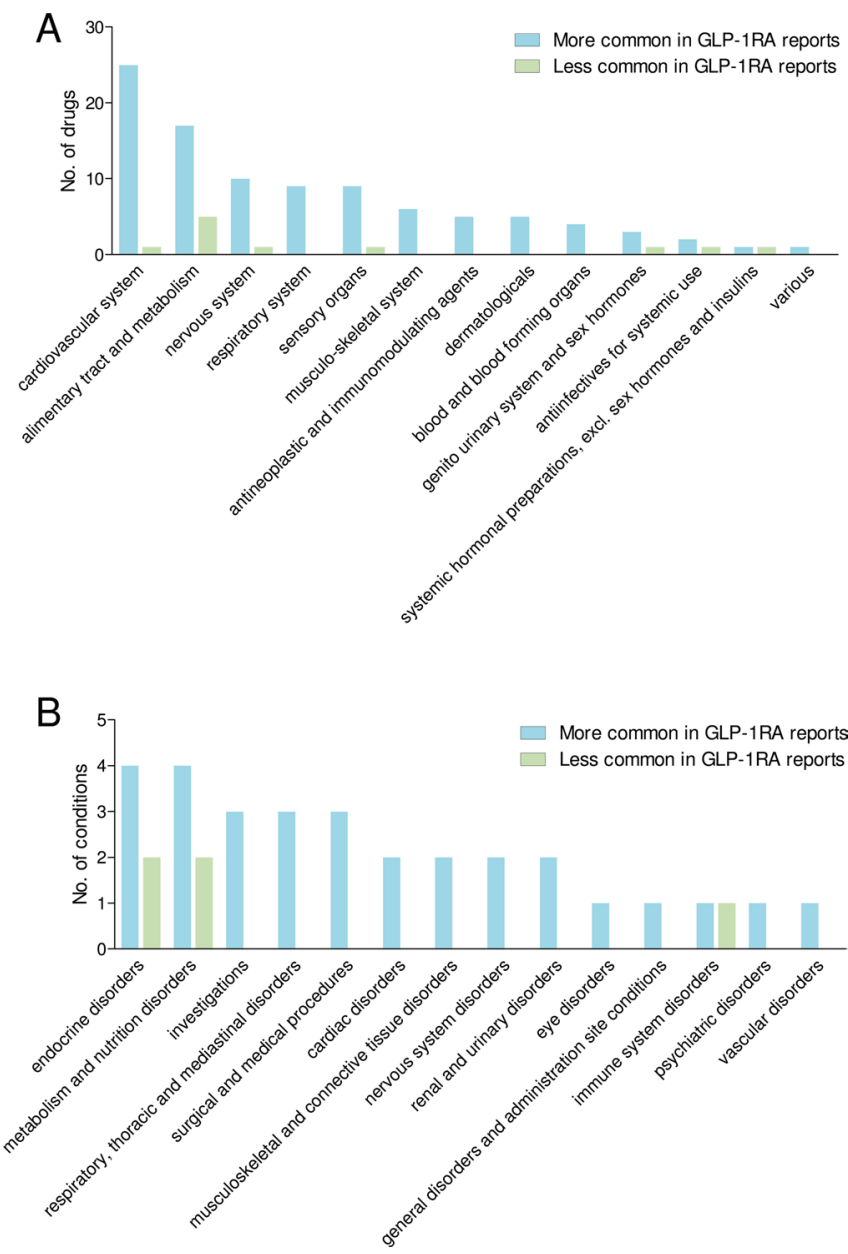

Figure 3 Comorbid conditions and concomitant medications. (A) Numbers of concomitant medications that were significantly more frequent or less frequent in reports with GLP-1RA or in reports with other GLMs. (B) Numbers of comorbid conditions (retrieved as indications for medications) that were significantly more frequent or less frequent in reports with GLP-1RA or in reports with other GLMs. GLMs, glucose-lowering medications; GLP-1RA, glucagon-like peptide-1 receptor agonists.

two most common GLP-1RAs in the FAERS, but was significantly lower for other GLP-1RAs pooled together, probably because of the overall low number of reports.

\section{DISCUSSION}

In this pharmacovigilance analysis of disproportionality, we found that GLP-1RAs are not associated with an increased reporting rate for retinal $\mathrm{AE}$, but rather with a significantly lower reporting ratio compared with other GLMs. Results are consistent across different models and subanalyses.

Together with the results of a recent meta-analysis, ${ }^{11}$ these data reassure the risk of retinopathy progression raised by the SUSTAIN-6 trial. ${ }^{3}$ In the absence of a specific drug-AE relation, we support the hypothesis that retinopathy progression in patients who received semaglutide in the SUSTAIN-6 trial may be attributed to the rapid improvement in glucose control, ${ }^{17}$ in insulin-treated patients with pre-existing retinopathy. ${ }^{18}$

Most specific retinal AEs were reported less frequently in association with GLP-1RA than other GLMs, with a few notable exceptions. Hemorrhagic retinopathy occurred more frequently in reports listing versus those not listing GLP-1RA, but this difference did not reach statistical significance and a quite similar and more common $\mathrm{AE}$ (retinal hemorrhage) occurred much less frequently and with high statistical significance in reports with GLP-1RA. The only $\mathrm{AE}$ listed significantly more often in reports with than in those without GLP-1RA was retinopexy, an intervention performed to repair retinal detachment, which can occur as a complication of advanced diabetic retinopathy. However, numbers were very small and retinal detachment occurred much less frequently and with high statistical significance in reports with GLP-1RA. Therefore, these data reasonably indicate that there was no consistent signal in the FAERS that GLP-1RA may be associated with AEs suggestive of diabetic retinopathy progression. For the evaluation of an emerging AE, drugs indicated as suspect or concomitant are considered equally because new signals may uncover for drugs not previously known to be responsible for such AE. Thus, the imbalanced distribution of suspect or concomitant drugs between GLP-1RA and other GLMs is irrelevant to the safety evaluation of retinal AE reports.

In the FAERS, up to $30 \%$ of reports listing GLM do not include a diabetes indication. This issue was noted previously, ${ }^{13} 19$ and may reflect that GLMs were being used for the treatment of pre-diabetes (eg, metformin and acarbose $)^{2021}$ or obesity (eg, liraglutide). ${ }^{22}$ However, incompleteness of reports likely contributes to the high number of reports listing GLM without a diabetes indication. Nonetheless, as retinopathy is a specific complication of diabetes, it is important to filter the diabetes indication to verify robustness of data. Vice versa, some reports $(2.5 \%)$ including diabetes as a concomitant condition may not list any GLM. Our data indicate that disproportionality of retinal AEs for GLP-1RA is not affected by the diabetes indication and associated GLM. Particularly, among GLMs, insulin therapy is typically considered as a proxy of disease severity, and retinopathy occurs more frequently in patients on insulin. ${ }^{23}$ Indeed, in the FAERS retinal AEs were more than four times more frequent in reports listing $(11.7 / 1000)$ than in those not listing insulin (2.9/1000). Importantly, retinal AEs were less frequently included in reports with GLP-1RAs than in those without, irrespective of concomitant insulin therapy.

It should be noted that lower rates of retinal AEs were associated with GLP-1RAs despite reports with GLP-1RAs listing more comorbid conditions and concomitant medications, including those for the treatment of hypertension, which is a risk factor for retinopathy progression. Therefore, the lower rate of retinal AEs is unlikely attributable to a lighter disease burden in GLP-1RA users. However, information on glucose and blood pressure 

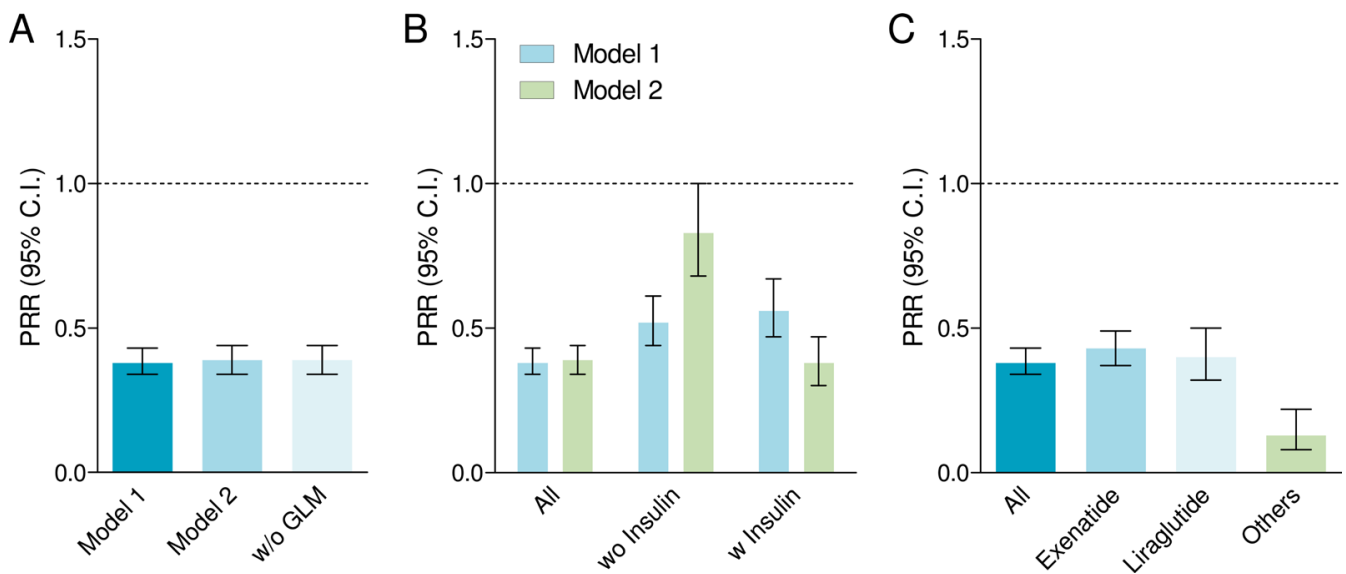

Figure 4 Results of sensitivity analyses. (A) The pooled PRRs for retinal AEs associated with GLP-1RAs resulting from sensitivity analysis (1), where reports were filtered by the diabetes indication irrespective of GLM, are compared with those of model 1 and model 2. (B) Pooled PRRs for retinal AEs associated with GLP-1RAs from sensitivity analysis (2), where reports including and those not including insulin are distinguished. (C) Pooled PRRs for retinal AEs associated with GLP-1RAs from sensitivity analysis (3), where exenatide, liraglutide, and other GLP-1RAs (albiglutide, dulaglutide, lixisenatide, and teduglutide) are examined separately. AE, adverse event; GLM, glucose-lowering medications; GLP-1RA, glucagon-like peptide-1 receptor agonists; PRR, proportional reporting ratio.

control, which are major determinants of retinopathy, is not available in pharmacovigilance databases. As a result, differences that likely exist between patients on GLP-1RA and those on other GLMs could not be adjusted for.

The FAERS does not yet contain AE reports for semaglutide, but the analysis by individual GLP-1RAs showed consistent results. Remarkably, no difference was noted between exenatide and liraglutide, which are two GLP-1RAs with very different chemical structures. The lower PRR associated with less common GLP-1RA likely reflects the small number of AEs filed for such drugs.

Several limitations of FAERS data have to be acknowledged. Above all, in most reports there is no demonstration of a causal relationship between drug exposure and the $\mathrm{AE}$, and some reports are filed by non-healthcare professionals. The inability to make causal inference is a limitation of all pharmacovigilance studies and it is shared by cohort observational studies. PRR in the FAERS does not inform on the true risk in clinical practice, because the population at risk is extensively under-represented. Furthermore, known drug AEs tend to be reported more frequently, thereby diluting new AE signals, and incompleteness of reports limits the emergence of links between concomitant conditions or drugs and new AEs.

Screening for retinopathy onset and progression should be performed routinely in patients with diabetes, but eventual drug-associated retinal AEs may not be captured in clinical practice as they are in clinical trials. In the FAERS, whether AE reports derived from clinical trials or routine pharmacovigilance cannot be reliably ascertained. Furthermore, there are no clear criteria to define whether a retinal event is to be considered part of the disease's natural history or a suspected adverse drug reaction. These difficulties may lead to an under-reporting of drug-associated retinal AEs.
Notwithstanding these limitations, pharmacovigilance assessment is an extremely helpful tool to monitor drug safety and detect new safety signals. There are several examples of how this can be applied to diabetes pharmacotherapy. For instance, the initial warning that sodium glucose cotransporter inhibitor 2 (SGLT2) inhibitors can cause diabetic ketoacidosis was primed by a disproportionality in the FAERS. ${ }^{24}$ Furthermore, FAERS analysis has recently confirmed that the SGLT2 inhibitor canagliflozin can increase the risk of lower extremity amputations. $^{13}$

In summary, our data indicate that, in the FAERS, retinal AEs are not disproportionally associated with GLP-1RA. As causal inference is impossible, we cannot conclude that GLP-1RA protects from retinopathy events, but these data are important from a safety perspective. Although we await for postmarketing information on semaglutide, our present study complements information originating from RCTs and reassures the risk of retinopathy progression associated with GLP-1RA therapy.

Contributors GPF designed the study, collected and analyzed the data, and wrote the manuscript. MS analyzed the data and revised the manuscript. AA designed the study and revised the manuscript.

Funding This research received no specific grant from any funding agency in the public, commercial or not-for-profit sectors.

Competing interests GPF reports grants, personal fees and non-financial support from AstraZeneca, personal fees and non-financial support from Boehringer Ingelheim, grants, personal fees and non-financial support from Eli Lilly, personal fees and non-financial support from Novo Nordisk, personal fees and nonfinancial support from Sanofi, non-financial support from Genzyme, personal fees and non-financial support from Abbott, personal fees and non-financial support from Novartis, and personal fees from Merck Sharp \& Dohme. MS reported no conflict of interest. AA reports grants, personal fees and non-financial support from AstraZeneca, personal fees from Boehringer Ingelheim, personal fees from Janssen, personal fees from Merck Sharp \& Dohme, personal fees and nonfinancial support from Novartis, personal fees from Sanofi, grants and personal fees from Mediolanum, personal fees from Novo Nordisk, personal fees from Lilly, 
personal fees and non-financial support from Servier, and personal fees from Takeda.

Provenance and peer review Not commissioned; externally peer reviewed. Data sharing statement Data on which the research is based are already publicly available in the FAERS, as specified in the Methods section.

Open Access This is an Open Access article distributed in accordance with the Creative Commons Attribution Non Commercial (CC BY-NC 4.0) license, which permits others to distribute, remix, adapt, build upon this work non-commercially, and license their derivative works on different terms, provided the original work is properly cited and the use is non-commercial. See: http://creativecommons.org/ licenses/by-nc/4.0/

(C) Article author(s) (or their employer(s) unless otherwise stated in the text of the article) 2018. All rights reserved. No commercial use is permitted unless otherwise expressly granted.

\section{REFERENCES}

1. Drucker DJ. The cardiovascular biology of glucagon-like peptide-1. Cell Metab 2016;24:15-30.

2. Marso SP, Daniels GH, Brown-Frandsen $\mathrm{K}$, et al. Liraglutide and cardiovascular outcomes in type 2 diabetes. $N$ Engl $\mathrm{J}$ Med 2016:375:311-22.

3. Marso SP, Bain SC, Consoli A, et al. Semaglutide and cardiovascular outcomes in patients with type 2 diabetes. N Engl J Med 2016;375:1834-44.

4. Holman RR, Bethel MA, Mentz RJ, et al. Effects of once-weekly exenatide on cardiovascular outcomes in type 2 diabetes. $N$ Engl $J$ Med 2017;377:1228-39.

5. Pfeffer MA, Claggett B, Diaz R, et al. Lixisenatide in patients with type 2 diabetes and acute coronary syndrome. N Engl J Med 2015;373:2247-57.

6. Mann JFE, Ørsted DD, Brown-Frandsen K, et al. Liraglutide and renal outcomes in type 2 diabetes. N Engl J Med 2017;377:839-48.

7. Varadhan L, Humphreys T, Walker AB, et al. The impact of improved glycaemic control with GLP-1 receptor agonist therapy on diabetic retinopathy. Diabetes Res Clin Pract 2014;103:e37-e39.

8. Simó R, Hernández C. GLP-1R as a target for the treatment of diabetic retinopathy: friend or foe? Diabetes 2017;66:1453-60.

9. Hernández C, Bogdanov P, Corraliza L, et al. Topical administration of GLP-1 receptor agonists prevents retinal neurodegeneration in experimental diabetes. Diabetes 2016;65:172-87.
10. Hernández C, Bogdanov P, Solà-Adell C, et al. Topical administration of DPP-IV inhibitors prevents retinal neurodegeneration in experimental diabetes. Diabetologia 2017;60:2285-98

11. Dicembrini I, Nreu B, Scatena A, et al. Microvascular effects of glucagon-like peptide-1 receptor agonists in type 2 diabetes: a meta-analysis of randomized controlled trials. Acta Diabetol 2017;54:933-41.

12. Patel A, Billot L, Reality BL. Reality and truth: balancing the hope and the hype of real-world evidence. Circulation 2017;136:260-2

13. Fadini GP, Avogaro A. SGTL2 inhibitors and amputations in the US FDA adverse event reporting system. Lancet Diabetes Endocrinol 2017;5:680-1.

14. Neal B, Perkovic V, Mahaffey KW, et al. Canagliflozin and cardiovascular and renal events in type 2 diabetes. $N$ Engl J Med 2017;377:644-57.

15. Almenoff JS, Pattishall EN, Gibbs TG, et al. Novel statistical tools for monitoring the safety of marketed drugs. Clin Pharmacol Ther 2007;82:157-66.

16. Sarangdhar M, Tabar S, Schmidt C, et al. Data mining differential clinical outcomes associated with drug regimens using adverse event reporting data. Nat Biotechnol 2016:34:697-700.

17. The Kroc Collaborative Study Group. Diabetic retinopathy after two years of intensified insulin treatment. Follow-up of the Kroc Collaborative Study. The Kroc Collaborative Study Group. JAMA 1988;260:37-41.

18. Vilsbøll T, Bain SC, Leiter LA, et al. Semaglutide, reduction in glycated haemoglobin and the risk of diabetic retinopathy. Diabetes Obes Metab 2017. 10.1111/dom.13172 (Epub ahead of print 27 Nov 2017).

19. Fadini GP, Bonora BM, Avogaro A. SGLT2 inhibitors and diabetic ketoacidosis: data from the FDA Adverse Event Reporting System. Diabetologia 2017;60:1385-9.

20. Perreault L, Kahn SE, Christophi CA, et al. Regression from prediabetes to normal glucose regulation in the diabetes prevention program. Diabetes Care 2009;32:1583-8.

21. Chiasson JL, Josse RG, Gomis R, et al. Acarbose for prevention of type 2 diabetes mellitus: the STOP-NIDDM randomised trial. Lancet 2002;359:2072-7.

22. Pi-Sunyer X, Astrup A, Fujioka $\mathrm{K}$, et al. A randomized, controlled trial of $3.0 \mathrm{mg}$ of liraglutide in weight management. $N$ Engl $J$ Med 2015;373:11-22.

23. Thomas RL, Dunstan F, Luzio SD, et al. Incidence of diabetic retinopathy in people with type 2 diabetes mellitus attending the Diabetic Retinopathy Screening Service for Wales: retrospective analysis. BMJ 2012;344:e874.

24. Bonora BM, Avogaro A, Fadini GP. Sodium-glucose co-transporter-2 inhibitors and diabetic ketoacidosis: An updated review of the literature. Diabetes Obes Metab 2018;20. 